\title{
Conocimiento de los riesgos del tabaquismo en fumadores, exfumadores y no fumadores
}

Enrique Ruiz Mori ${ }^{1}$, Hernán Ruiz Mori ${ }^{1}$, Ronald Salazar-Rojas ${ }^{2}$, Cristina Torres-Mallma ${ }^{2}$, Sergio Valer-Villanueva ${ }^{2,3}$, Brendy Santiago ${ }^{2,3}$, Humberto Joel Blas-Rodriguez ${ }^{2}$, Cindy Vásquez Tornero ${ }^{2}$, Leslye Guevara Gonzales ${ }^{2,3}$

\section{RESUMEN}

Objetivo: Determinar en una población de no fumadores, fumadores y exfumadores, el nivel de conocimiento sobre los peligros y riesgos en la salud que genera el tabaquismo.

Material y Métodos: Es un estudio epidemiológico, observacional, descriptivo y transversal, que se realizó en setiembre del 2015 en la ciudad de Lima y el Callao. Se aplicó una encuesta a mayores de 18 años. Los participantes fueron divididos en tres grupos, fumadores, exfumadores y no-fumadores.

Resultados: Se incluyeron a 2270 sujetos, 744 eran fumadores, 752 exfumadores y 774 no- fumadores. El grupo que más asoció el tabaco a enfermedades es el de exfumadores, el 53,8\% de ellos mencionaban al cáncer de pulmón. El grupo de nofumadores fue el que con menos frecuencia conocía los peligros del tabaquismo. En los tres grupos: la enfermedad más relacionada al tabaco fue el cáncer de pulmón, seguida por el infarto del miocardio, mientras que la fertilidad se asoció poco. La televisión fue el principal medio de difusión sobre los peligros del fumar, mientras las redes sociales aún no tienen un rol preponderante. A los ex- fumadores les impactó más la información sobre los riesgos del hábito de fumar ( $p<0.05)$.

Conclusión: Los exfumadores son los que tenían mayor información sobre los peligros del tabaquismo en la salud. Los tres grupos relacionaban más al tabaco con el cáncer de pulmón, y existía poca información sobre la fertilidad y el consumo del cigarrillo. A los exfumadores les impactó más la información antitabáquica. La televisión fue el principal medio de difusión sobre los peligros del fumar.

Palabras Clave: Tabaquismo, consumo de tabaco, conocimiento del tabaco, educación para la salud, riesgo cardiovascular.

\section{Knowledge of risk tobacco in smokers, former-smokers and non-smokers}

\section{ABSTRACT}

Objetive: Determine in a population of non-smokers, smokers and former-smokers, the level of knowledge of the health risks that smoking generate.

Material and Methods: An epidemiological, observational, descriptive and cross-sectional research, was conducted in September 2015 in the city of Lima and Callao. A survey of over 18 was applied. Participants were divided into three groups, smokers, former-smokers and non-smokers.

Results: The study included 2270 subjects, 744 were smokers, 752 former-smokers and 774 non-smokers. The group that mostly associated the tobacco to many diseases was the group of former-smokers, $53.8 \%$ of them mentioned to lung cancer as the most common disease related to tobacco. The non-smokers was the group that knows less often the risks of smoking. In all three groups, the most tobacco-related disease was the lung cancer, followed by myocardial infarction, while fertility was little associated. Television was the main means of dissemination about the dangers of smoking, while social networks do not have a leading role. For the former-smokers will hit more information about the risks of smoking $(p<0.05)$.

Conclusion: Former-smokers had more information on the risk of smoking. In the three groups the most tobacco-related disease was the lung cancer, and there was very little information about fertility and cigarette consumption. Formersmokers do the impacted more risk information cigarette. Television remains the main instrument to fight against smoking.

Keywords: Smoking, Tobacco consumption, Knowledge of tobacco, Health promotion, Cardiovascular Risk

\footnotetext{
${ }^{1}$ Profesor de Fisiología y Fisiopatología. Facultad de Medicina Universidad de San Martín de Porres.

2 Alumnos de la Facultad de Medicina USMP.

${ }^{3}$ Sociedad de Estudiantes de Medicina de la Universidad de San Martín de Porres (SOCIEM-USMP)
} 


\section{INTRODUCCIÓN}

El tabaco es la segunda droga más consumida a nivel mundial luego del alcohol, a pesar de ser legal existen múltiples evidencias que relacionan al tabaquismo con más de 25 enfermedades tales como el cáncer de pulmón, cáncer de laringe, enfisema pulmonar, infarto cardiaco, problemas en la fertilidad, menopausia prematura, entre otras. Pero sus efectos no sólo se presentan en el fumador, sino también en los denominados fumadores pasivos, que aspiran el humo de "segunda mano" y ponen en riesgo su salud $(1,2)$.

El informe de la OMS de julio del 2015, muestra que a nivel mundial existen aproximadamente 1200 millones de personas que fuman y que el $80 \%$ de ellos viven en países de ingresos bajos o medios, donde es mayor la carga de morbilidad y mortalidad asociada al tabaquismo (3). Es preocupante que cada año el fumar cigarrillos genere alrededor de 6 millones de muertes en el mundo, de las cuales más de 5 millones son de consumidores directos y más de 600 mil son de fumadores pasivos. De mantenerse las tendencias actuales, el consumo de tabaco ocasionará la muerte de más de ocho millones de personas en el año 2030. En Latino-América según el estudio INTERHEART el tabaco es el segundo factor de riesgo coronario y se estima que produce alrededor de quinientas mil muertes $(4,5)$.

La lucha antitabáquica incluye mecanismos para disminuir tanto la oferta como la demanda del tabaco, aplica políticas que buscan que los jóvenes no se inicien en el consumo, protege a los no fumadores de la exposición al humo de segunda mano, ayuda a los consumidores a abandonar el cigarrillo y a los exfumadores a mantener la abstinencia $(6,7)$.

Los esfuerzos que nuestra sociedad está realizando en la lucha contra el tabaco a través de entidades gubernamentales y no gubernamentales están dando resultados positivos, un ejemplo de ello son los estudios TORNASOL I y II realizados en el Perú los años 2004 y 2009 respectivamente. En dichos estudios se demostró que en ese lapso de tiempo la prevalencia del tabaquismo disminuyó en el país de $26.1 \%$ a $23.2 \%$, cifras que expresan que sí se puede actuar a través de programas adecuadamente estructurados sobre los estilos de vida desfavorables que inundan cada vez más a las ciudades que crecen económicamente $(8,9)$.

La información y sobre todo la educación de los peligros que encierra el tabaquismo en la comunidad, se convierte en una herramienta muy útil y fundamental para lograr la prevención y el control del hábito de fumar. Si bien las múltiples campañas publicitarias sobre los riesgos de fumar en la salud impactan en la población, es necesario evaluar el nivel de conocimiento en la colectividad.

Por ello el objetivo del presente estudio es determinar en una población de no fumadores, fumadores y exfumadores, el nivel de conocimiento sobre los peligros y riesgos en la salud que genera el tabaquismo y si ello influye en la conducta del fumador.

\section{MATERIAL Y MÉTODOS}

Es un estudio epidemiológico, observacional, descriptivo y transversal en el que se analizó el conocimiento de los riesgos del tabaquismo en la salud. Fue realizado en el mes de setiembre del 2015 en la ciudad de Lima y la Provincia Constitucional del Callao; en donde se aplicó una encuesta validada a través de una prueba piloto en 20 sujetos y asesorado por un comité de expertos en tabaquismo. Los criterios de inclusión fueron: ser mayor de 18 años y que aceptaran participar en un estudio sobre tabaquismo voluntariamente firmando el respectivo consentimiento informado y consignando su número telefónico.

Los participantes fueron divididos en tres grupos, dependiendo de su condición, uno de fumadores, otro de exfumadores y el tercero de no fumadores. Según la clasificación estándar de la OMS se definió fumador a aquella persona que ha fumado por lo menos un cigarrillo en los últimos 6 meses, exfumador a la persona que habiendo sido fumador se ha mantenido en abstinencia al menos por los últimos 6 meses, y no fumador a la persona que nunca ha fumado o ha fumado menos de 100 cigarrillos en toda su vida (10).

En la recolección de datos, participaron 380 alumnos del tercer año de medicina de la Universidad de San Martín de Porres; y se mantuvo total confidencialidad de la información obtenida.

Los datos obtenidos a partir de la encuesta fueron tabulados en el programa Microsoft Excel 2013 y luego exportados al software SPSS 22.

\section{RESULTADOS}

Por contar con la información completa de sus respectivos cuestionarios, se incluyeron a 2,270 sujetos de los 2,390 encuestados. 744 participantes eran fumadores, 752 exfumadores y 774 no fumadores. 
Tabla 1. Característica de la población según Género

\begin{tabular}{|ccccc|}
\hline & Fumadores & No Fumadores & Ex fumadores & Total \\
\hline Masculino & $391(34,5 \%)$ & $323(28,5 \%)$ & $419(37,0 \%)$ & $1133(100 \%)$ \\
\hline Femenino & $353(31,0 \%)$ & $451(39,7 \%)$ & $333(29,3 \%)$ & $1137(100 \%)$ \\
\hline
\end{tabular}

El porcentaje de varones que fuman es ligeramente predominaban los varones (Tabla 1). mayor que las mujeres. En el grupo de exfumadores

Tabla 2 Característica de la población por grupo etario y género

\begin{tabular}{|c|c|c|c|c|c|c|c|c|c|}
\hline Edad & \multicolumn{3}{|c|}{ Fumadores } & \multicolumn{3}{|c|}{ No fumadores } & \multicolumn{3}{|c|}{ Ex fumadores } \\
\hline \multirow{2}{*}{ Años } & \multicolumn{2}{|c|}{ Género } & \multirow{2}{*}{ Total } & \multicolumn{2}{|c|}{ Género } & \multirow{2}{*}{ Total } & \multicolumn{2}{|c|}{ Género } & \multirow{2}{*}{ Total } \\
\hline & M & $\mathrm{F}$ & & $M$ & $\mathrm{~F}$ & & M & $\mathrm{F}$ & \\
\hline$<20$ & $\begin{array}{c}74 \\
(49,0 \%)\end{array}$ & $\begin{array}{c}77 \\
(51,0 \%)\end{array}$ & $\begin{array}{c}151 \\
(20,3 \%)\end{array}$ & $\begin{array}{c}80 \\
(55,9 \%)\end{array}$ & $\begin{array}{c}63 \\
(44,1 \%)\end{array}$ & $\begin{array}{c}143 \\
(18,5 \%)\end{array}$ & $\begin{array}{c}49 \\
(54,4 \%)\end{array}$ & $\begin{array}{c}41 \\
(45,6 \%)\end{array}$ & $\begin{array}{c}90 \\
(12,0 \%)\end{array}$ \\
\hline $20-29$ & $\begin{array}{c}245 \\
(53,0 \%)\end{array}$ & $\begin{array}{c}217 \\
(47,0 \%)\end{array}$ & $\begin{array}{c}462 \\
(62,1 \%)\end{array}$ & $\begin{array}{c}209 \\
(60,1 \%)\end{array}$ & $\begin{array}{c}139 \\
(39,9 \%)\end{array}$ & $\begin{array}{c}348 \\
(45,0 \%)\end{array}$ & $\begin{array}{c}169 \\
(57,5 \%)\end{array}$ & $\begin{array}{c}125 \\
(42,5 \%)\end{array}$ & $\begin{array}{c}294 \\
(39,1 \%)\end{array}$ \\
\hline $30-39$ & $\begin{array}{c}28 \\
(65,1 \%)\end{array}$ & $\begin{array}{c}15 \\
(34,9 \%)\end{array}$ & $\begin{array}{c}43 \\
(5,8 \%)\end{array}$ & $\begin{array}{c}31 \\
(62,0 \%)\end{array}$ & $\begin{array}{c}19 \\
(38,0 \%)\end{array}$ & $\begin{array}{c}50 \\
(6,5 \%)\end{array}$ & $\begin{array}{c}32 \\
(52,5 \%)\end{array}$ & $\begin{array}{c}29 \\
(47,5 \%)\end{array}$ & $\begin{array}{c}61 \\
(8,1 \%)\end{array}$ \\
\hline $40-49$ & $\begin{array}{c}16 \\
(45,7 \%)\end{array}$ & $\begin{array}{c}19 \\
(54,3 \%)\end{array}$ & $\begin{array}{c}35 \\
(4,7 \%)\end{array}$ & $\begin{array}{c}38 \\
(59,4 \%)\end{array}$ & $\begin{array}{c}26 \\
(40,6 \%)\end{array}$ & $\begin{array}{c}64 \\
(8,3 \%)\end{array}$ & $\begin{array}{c}55 \\
(52,4 \%)\end{array}$ & $\begin{array}{c}50 \\
(47,6 \%)\end{array}$ & $\begin{array}{c}105 \\
(14,0 \%)\end{array}$ \\
\hline $50-59$ & $\begin{array}{c}19 \\
(47,5 \%)\end{array}$ & $\begin{array}{c}21 \\
(52,5 \%)\end{array}$ & $\begin{array}{c}40 \\
(5,4 \%)\end{array}$ & $\begin{array}{c}43 \\
(48,3 \%)\end{array}$ & $\begin{array}{c}46 \\
(51,7 \%)\end{array}$ & $\begin{array}{c}89 \\
(11,5 \%)\end{array}$ & $\begin{array}{c}53 \\
(51,5 \%)\end{array}$ & $\begin{array}{c}50 \\
(48,5 \%)\end{array}$ & $\begin{array}{c}103 \\
(13,7 \%)\end{array}$ \\
\hline$>59$ & $\begin{array}{c}9 \\
(69,2 \%)\end{array}$ & $\begin{array}{c}4 \\
(30,8 \%)\end{array}$ & $\begin{array}{c}13 \\
(1,7 \%)\end{array}$ & $\begin{array}{c}50 \\
(62,5 \%)\end{array}$ & $\begin{array}{c}30 \\
(37,5 \%)\end{array}$ & $\begin{array}{c}80 \\
(10,3 \%)\end{array}$ & $\begin{array}{c}61 \\
(61,6 \%)\end{array}$ & $\begin{array}{c}38 \\
(38,4 \%)\end{array}$ & $\begin{array}{c}99 \\
(13,2 \%)\end{array}$ \\
\hline
\end{tabular}

En la Tabla 2 se observa que en los tres grupos predominó la población de 20 a 29 años, donde la

mayor presencia fueron los varones. El segundo grupo etario en frecuencia fueron los menores de 20 años

Tabla 3. Conocimiento de enfermedades relacionadas al tabaquismo

\begin{tabular}{|c|c|c|c|c|c|c|c|c|c|}
\hline \multirow{3}{*}{ Conocimiento } & \multicolumn{3}{|c|}{ Fumadores } & \multicolumn{3}{|c|}{ No fumadores } & \multicolumn{3}{|c|}{ Ex Fumadores } \\
\hline & \multicolumn{2}{|c|}{ Género } & \multirow{2}{*}{ Total } & \multicolumn{2}{|c|}{ Género } & \multirow{2}{*}{ Total } & \multicolumn{2}{|c|}{ Género } & \multirow{2}{*}{ Total } \\
\hline & $\mathrm{F}$ & $\mathrm{M}$ & & $\mathrm{F}$ & M & & $\mathrm{F}$ & $M$ & \\
\hline Cáncer de Pulmón & $\begin{array}{c}17 \\
3\end{array}$ & $\begin{array}{c}18 \\
7\end{array}$ & $\begin{array}{c}360 \\
(48,3 \%)\end{array}$ & 116 & $\begin{array}{c}17 \\
0\end{array}$ & $\begin{array}{c}286 \\
(36,9 \%)\end{array}$ & $\begin{array}{c}18 \\
3\end{array}$ & $\begin{array}{c}22 \\
2\end{array}$ & $\begin{array}{c}405 \\
(53,8 \%)\end{array}$ \\
\hline $\begin{array}{l}\text { Infarto al } \\
\text { Miocardio }\end{array}$ & 79 & 72 & $\begin{array}{c}151 \\
(20,3 \%)\end{array}$ & 40 & 66 & $\begin{array}{c}106 \\
(13,6 \%)\end{array}$ & 54 & 83 & $\begin{array}{c}137 \\
(18,2 \%)\end{array}$ \\
\hline $\begin{array}{l}\text { Enfisema } \\
\text { Pulmonar }\end{array}$ & 58 & 69 & $\begin{array}{c}127 \\
(17,0 \%)\end{array}$ & 37 & 58 & $\begin{array}{c}95 \\
(12,2 \%)\end{array}$ & 46 & 62 & $\begin{array}{c}108 \\
(14,3 \%)\end{array}$ \\
\hline Impotencia Sexual & 43 & 63 & $\begin{array}{c}106 \\
(14,2 \%)\end{array}$ & 28 & 46 & $\begin{array}{c}74 \\
(9,5 \%)\end{array}$ & 30 & 57 & $\begin{array}{c}87 \\
(11,5 \%)\end{array}$ \\
\hline
\end{tabular}

En la tabla 3, se aprecia que el grupo que más asocia el tabaquismo con enfermedades es de los exfumadores, mientras que el grupo de no fumadores es que menos lo relaciona. En los tres grupos los varones registran mayor conocimiento de los peligros del tabaquismo en comparación con las mujeres. La enfermedad que más asocian al cigarrillo es el cáncer de pulmón, seguido por el infarto al miocardio; mientras que los problemas de impotencia sexual es el menos mencionado. 
Tabla 4. Fuente de información

\begin{tabular}{|cccc|}
\hline Medio de Información & Fumadores & No fumadores & Ex fumadores \\
\hline Ninguno & $26(3,5 \%)$ & $28(3,62)$ & $8(1,1 \%)$ \\
\hline TV & $549(73,8 \%)$ & $473(61,11)$ & $504(67,0 \%)$ \\
\hline Periódico & $302(40,6 \%)$ & $368(47,55)$ & $333(44,3 \%)$ \\
\hline Revista & $281(37,8 \%)$ & $300(38,76)$ & $260(34,6 \%)$ \\
\hline Campañas & $376(50,5 \%)$ & $430(55,56)$ & $336(44,7 \%)$ \\
\hline Colegio & $460(61,8 \%)$ & $346(44,70)$ & $332(44,1 \%)$ \\
\hline Universidad & $394(53,0 \%)$ & $353(44,32)$ & $298(39,6 \%)$ \\
\hline Folletos & $284(38,2 \%)$ & $316(40,83)$ & $198(26,3 \%)$ \\
\hline Internet & $399(53,6 \%)$ & $386(49,87)$ & $317(42,2 \%)$ \\
\hline YouTube & $182(24,5 \%)$ & $234(30,23)$ & $156(20,7 \%)$ \\
\hline Facebook & $264(35,5 \%)$ & $253(32,69)$ & $204(27,1 \%)$ \\
\hline Otros & & $117(15,12)$ & \\
\hline
\end{tabular}

Según la tabla 4, el principal medio de información sobre los riesgos que implica el tabaquismo en los tres grupos fue la televisión. En segundo lugar en el grupo de fumadores fue la educación impartida en colegios y universidades, en tanto que en el grupo de los no fumadores las campañas educativas de prevenczión eran la segunda opción; en el grupo de exfumadores prácticamente las campañas educativas, los periódicos y el colegio son el segundo medio de difusión. También se puede apreciar que en los tres grupos las redes sociales de comunicación no tienen un rol preponderante.

Tabla 5. Impacto del conocimiento del tabaquismo por género.

\begin{tabular}{|c|c|c|c|c|c|c|c|c|c|}
\hline \multirow{3}{*}{ Impactó } & \multicolumn{3}{|c|}{ Fumadores } & \multicolumn{3}{|c|}{ No fumadores } & \multicolumn{3}{|c|}{ Ex fumadores } \\
\hline & \multicolumn{2}{|c|}{ Género } & \multirow{2}{*}{ Total } & \multicolumn{2}{|c|}{ Género } & \multirow{2}{*}{ Total } & \multicolumn{2}{|c|}{ Género } & \multirow{2}{*}{ Total } \\
\hline & $M$ & $\mathrm{~F}$ & & M & $\mathrm{F}$ & & M & $\mathrm{F}$ & \\
\hline No & $\begin{array}{c}133 \\
(45,4 \%)\end{array}$ & $\begin{array}{c}133 \\
(54,6 \%)\end{array}$ & $\begin{array}{c}293 \\
(39,4 \%)\end{array}$ & $\begin{array}{c}129 \\
(59,4 \%)\end{array}$ & $\begin{array}{c}88 \\
(40,6 \%)\end{array}$ & $\begin{array}{c}217 \\
(28,0 \%)\end{array}$ & $\begin{array}{c}74 \\
(47,1 \%)\end{array}$ & $\begin{array}{c}83 \\
(52,9 \%)\end{array}$ & $\begin{array}{c}157 \\
(20,9 \%)\end{array}$ \\
\hline Si & $\begin{array}{c}220 \\
(48,8 \%)\end{array}$ & $\begin{array}{c}231 \\
(51,2 \%)\end{array}$ & $\begin{array}{c}451 \\
(60,6 \%)\end{array}$ & $\begin{array}{c}322 \\
(57,8 \%)\end{array}$ & $\begin{array}{c}235 \\
(42,2 \%)\end{array}$ & $\begin{array}{c}557 \\
(72,0 \%)\end{array}$ & $\begin{array}{c}259 \\
(43,5 \%)\end{array}$ & $\begin{array}{c}336 \\
(22,3 \%)\end{array}$ & $\begin{array}{c}595 \\
(79,1 \%)\end{array}$ \\
\hline
\end{tabular}

${ }^{*}$ Condición de exfumador $\mathrm{p}<0.05$

En relación al impacto del conocimiento de los peligros que conlleva el hábito del tabaquismo, en el grupo de exfumadores el impacto fue mayor $(p<$ $0.05)$. En los tres grupos a los varones les impactó más

\section{DISCUSIÓN}

En los últimos años, la lucha antitabáquica está alcanzando importantes logros a través de múltiples campañas educativas dirigidas a diferentes áreas de la comunidad, pero la efectividad de estas intervenciones se ve reflejado en el conocimiento que adquiere finalmente la población.

Diversos trabajos han propuesto que el conocimiento por parte de los adolescentes sobre los riesgos que la información del daño que puede producir el cigarrillo. El grupo de fumadores es el que alcanzó el menor número de sensibilizados (Tabla 5).

existe en el cigarrillo es uno de los motivos principales por los que condiciona que muchos de ellos no se inicien en el tabaquismo. En el estudio realizado a los no fumadores, sobre todo a los varones, les impactó más la información sobre los peligros del cigarrillo, probablemente esto evitaría que se inicien en el consumo. Sin embargo el enfoque que se puede hacer en relación al riesgo de fumar no es la adecuada, así muchas veces la percepción de los riesgos a largo plazo como son las enfermedades del corazón o del cáncer de pulmón, deben combinarse 
con otros riesgos más cercanos a esta población juvenil como son los problemas de fertilidad en la mujer o de impotencia en el varón, cuyo conocimiento es bajo y se ve reflejado en los resultados del estudio (11).

La relación de tabaquismo y fertilidad no es muy promocionada, por lo que el nivel de conocimiento del cigarrillo y la función sexual es muy baja. Este hecho cobra mucha importancia al tener en cuenta que los jóvenes que empiezan a fumar y reciben información del daño del tabaco que generalmente está dirigido al cáncer o al infarto, patologías que son poco frecuentes en este grupo etario, por lo que pueden deducir erróneamente que son peligros en el largo plazo; mientras que si se diera a conocer más los efectos del cigarrillo en la disminución de la función sexual del varón e infertilidad en la mujer, dos hechos claramente demostrados en diversos estudios clínicos, probablemente propiciaría un menor inicio temprano del consumo del cigarrillo.

Generalmente para adquirir la condición de exfumador el sujeto debe haber deteriorado su salud en forma considerable por un diagnóstico de cáncer, infarto agudo de miocardio o haber experimentado esta situación en un familiar o amistad muy cercana (12). En el estudio se logra evidenciar que los exfumadores tendrían mayor información de los peligros del tabaquismo, lo que influenciaría en cierto grado al fumador en abandonar su hábito en comparación con los sujetos que siguen fumando al no tener el conocimiento o la convicción de lo dañino que es el tabaco. Así mismo en la literatura se describen trabajos que muestran a los exfumadores y a los fumadores pasivos conocer más sobre los efectos del tabaquismo en la salud (13), por ello es necesario también ahondar la investigación en los fumadores de "segunda mano", a fin de contar con una nueva herramienta que sirva de forma eficaz en la lucha contra el tabaco.

El nivel de conocimiento de los peligros del tabaquismo en el fumador son bajos, los mitos y los conceptos erróneos sobre el cigarrillo hace que los fumadores se mantengan activos en su uso $(14,15)$. En el presente estudio un alto porcentaje de los fumadores conocen los peligros del fumar, sobre todo la relación de cáncer y tabaco, sin embargo no abandonan el cigarrillo mostrando la falta de convicción. Este resultado es muy semejante a diversas publicaciones que asocian el hábito tabáquico al cáncer de pulmón y consideran al alquitrán como el componente más tóxico del humo de tabaco (16).
Llama la atención que en el estudio las redes sociales no están siendo el principal medio de difusión, teniendo en cuenta que en los últimos años se ha desarrollado vertiginosamente el uso del internet, así el Twitter o el Facebook cuenta con más de 100 millones de usuarios mensuales cada uno, y siendo un sistema abierto y en construcción permanente involucra rápidamente necesidades y problemas dirigidos a personas, grupos e instituciones (17). Sin embargo en nuestro medio, la televisión aún sigue siendo la principal herramienta de comunicación, esto debe plantear un nuevo enfoque considerando que la juventud utiliza en forma permanente los medios digitales, que tienen un bajo costo y una alta difusión.

El estudio tiene la limitación de haberse hecho en una población citadina, por lo que los resultados no son extrapolables a una población rural.

En conclusión, el grupo de exfumadores es el que tenía mayor información sobre los peligros del tabaquismo en la salud. Los tres grupos relacionaban más al tabaco con el cáncer de pulmón, y existía muy poca información sobre la fertilidad y el consumo del cigarrillo. A los exfumadores les impactó más la información antitabáquica. La televisión fue el principal medio de difusión sobre los peligros del fumar.

Se recomienda que se debería utilizar más las redes sociales para la educación de la comunidad sobre los peligros del tabaquismo, especialmente en los jóvenes.

\section{REFERENCIAS BIBLIOGRÁFICAS}

1. U.S. Department of Health and Human Services. The Health Consequences of Smoking: A Report of the Surgeon General. Atlanta: Department of Health and Human Services, Centers for Disease Control and Prevention, National Center for Chronic Disease Prevention and Health Promotion, Office on Smoking and Health, 2004.

2. Bello S, Michalland S, Soto M, Contreras C, Salinas J. Efectos de la exposición al humo de tabaco ambiental en no fumadores. Rev Chil Enf Respir 2005; 21: 179-192

3. Organización Mundial de la Salud. Tabaco. Nota descriptiva $N^{\circ}$ 339. Ginebra. Julio 2015.

4. Lanas F, Avezum A, Bautista LE, Diaz R, Luna M, Islam S, et al. INTERHEART Investigators in Latin America. Risk factors for acute myocardial infarction in Latin America: the INTERHEART Latin American study. Circulation. 2007;115:1067-1074.

5. Lanas F, Toro V, Cortés R, Sánchez A. INTERHEART, un estudio de casos y controles sobre factores de riesgo de infarto del 
Enrique Ruiz Mori, Hernán Ruiz Mori, Ronald Salazar-Rojas, Cristina Torres-Mallma, Sergio Valer-Villanueva, Brendy Santiago, Humberto Joel Blas-Rodriguez, Cindy Vásquez Tornero, Leslye Guevara Gonzales

miocardio en el mundo y América Latina. Med UIS. 2009;21:176-182.

6. Hernández J, Guevara L, García M, Tascón J. Hábito de Fumar en los Estudiantes de Primeros Semestres de la Facultad de Salud: Características y Percepciones. Colombia Médica. 2006; 37(1):31-38.

7. Salmerón-C J, Arillo-S E, Campuzano-R. J C, López-A F, Lascano-P. E. Tabaquismo en profesionales de la salud del Instituto Mexicano del Seguro Social. Salud Pública. MEX. 2002, 44 sup. 1 : S 67 - S 75.

8. Segura L, Agusti R, Ruiz E. Factores de Riesgo de las Enfermedades Cardiovasculares en el Perú II. Estudio TORNASOL II comparado con Tornasol I después de cinco años. Rev Per Cardiol. 2013;1:5-59.

9. Ruiz E. Riesgo y Prevención Cardiovascular. $1^{\circ}$ Ed. Lima, 2014.

10. Colegio Médico del Perú. Guía Nacional de abordaje técnico al Tabaquismo. Perú; 2010.

11. Rodriguez D, Romer D, Audrain-McGovern J. Beliefs about the risks of smoking mediate the relationship between exposure to smoking and smoking. Psychosom Med 2007;69(1):106-113.

12. Ruiz E, Ayala L, Burgos J. Programas educativos antitabaco y su relación con el hábito de fumar. Acta Cancerológica 2013; 42 (1): 17-25.

13. Sonzg A, , Morrell H, , Cornell J, Ramos M, Biehl M, Kropp R,el at. Perceptions of Smoking-Related Risks and Benefits as Predictors of Adolescent Smoking Initiation. Am J Public Health. 2009 March; 99(3): 487-492.

14. Pinilla-Vásquez C, Angarita-Fonseca A, Conocimientos y actitudes asociadas al inicio del hábito de fumar durante la vida universitaria. Revista hacia la promoción de la salud. 2012;17(2):25-39.

15. Festner J. Conocimientos, actitudes y prácticas sobre el tabaquismo en estudiantes de Enfermería y Obstetricia del Instituto “Dr. Andrés Barbero" Año 2007. Mem Inst Investig Cienc Salud. 2008;6(2):38-47

16. León $\mathrm{D}$, Ferreira P, Pillon $\mathrm{S}$. Conocimientos y prácticas sobre el consumo de tabaco en estudiantes de pregrado de farmacia, Lima, Perú. Rev Latino-Am. 2010;18:582-8.

17. Hernández T, Ocampo M, Sabido R. Redes sociales de internet en difusión antitabáquica: la experiencia de la clínica contra el tabaquismo del Hospital General de México “Eduardo Liceaga”. Gaceta Médica de México. 2012;148:438-47.

\section{Fuentes de Financiamiento}

Este artículo ha sido financiado por los autores.

\section{Conflicto de interés}

Los autores declaran no tener ningún conflicto de interés.

\section{Correspondencia:}

Enrique Ruiz Mori

Dirección: Av. El corregidor 1531 - Las Viñas - La Molina

Teléfono: 3652300 - 182

Correo electrónico: cruizm@usmp.pe 